ED-based youth violence intervention planned for the summer of 2010.

\section{USING MIXED METHODS RESEARCH TO DEVELOP AN EMERGENCY DEPARTMENT-BASED YOUTH VIOLENCE SECONDARY INTERVENTION}

C Snider*, A Nathens Correspondence: Li Ka Shing Knowledge Institute, St. Michael's Hospital, University of Toronto, Emergency Department 30 Bond Street, Room 1-008 Shuter Wing Toronto, ON M5B 1W8, Canada

10.1136/ip.2010.029215.670

Violence is a significant cause of morbidity and mortality among youth worldwide. Using mixed methods is essential to understanding the complexity of addressing youth violence. Emergency Department (ED)-based interventions that target a high risk group are ideal because victims of violence are more likely to become repeat victims of violence. We used mixed methods research to develop an ED-based youth violence secondary intervention in Toronto, Canada. To determine where best to link the patient and program, we conducted quantitative studies using the population-based National Ambulatory Care Reporting System. Our first study demonstrated that focusing on admitted patients would miss most opportunities for intervention. Our second study examined the type of ED that would be most appropriate. While most efforts at secondary violence prevention target patients cared for at designated trauma centres, our work suggests that opportunities are greater outside these centres. We then performed a systematic review of ED-based youth violence secondary prevention programs. Finally, to ensure the best design of the intervention and to build important partnerships, we engaged in community-based participatory research with over 100 youth, parents and youth violence community workers. Using concept mapping we worked with community partners to develop a program that will link youth who visit the ED with injuries due to violence with community youth violence interventions. We will discuss the results of the above studies and share preliminary results from the pilot project of the 